\title{
Cryptanalysis of a code-based one-time signature
}

\author{
Jean-Christophe Deneuville • Philippe \\ Gaborit
}

Received: date / Accepted: date

\begin{abstract}
In 2012, Lyubashevsky introduced a new framework for building latticebased signature schemes without resorting to any trapdoor (such as GPV [6] or NTRU [7]). The idea is to sample a set of short lattice elements and construct the public key as a Short Integer Solution (SIS for short) instance. Signatures are obtained using a small subset sum of the secret key, hidden by a (large) Gaussian mask. (Information leakage is dealt with using rejection sampling.) Recently, Persichetti proposed an efficient adaptation of this framework to coding theory [12]. In this paper, we show that this adaptation cannot be secure, even for one-time signatures (OTS), due to an inherent difference between bounds in Hamming and Euclidean metrics. The attack consists in rewriting a signature as a noisy syndrome decoding problem, which can be handled efficiently using the extended bit flipping decoding algorithm. We illustrate our results by breaking Persichetti's OTS scheme built upon this approach [12]: using a single signature, we recover the secret (signing) key in about the same amount of time as required for a couple of signature verifications.
\end{abstract}

Keywords Post-Quantum Cryptography · Coding Theory · Signature · Cryptanalysis · MSC 94A60, 11T71, 14G50

\section{Introduction}

Building efficient and secure full-time (stateless) signature schemes from coding theory assumptions is a long standing open problem. Few years ago, Lyubashevsky proposed a new method for obtaining digital signatures from lattice assumptions, that does not require the use of a trapdoor [8]. This method follows the baseline of Pointcheval-Stern [13]. The construction works by sampling relatively short

J.-C. Deneuville

École Nationale de l'Aviation Civile, Federal University of Toulouse, France

E-mail: jean-christophe.deneuville@enac.fr

P. Gaborit

XLIM-MATHIS, University of Limoges, France

E-mail: philippe.gaborit@unilim.fr 
lattice vectors, used as the secret key. The public key is an instance of the SIS problem. To produce a digital signature, the signer commits a masking value, receives a challenge depending on the message to sign and the committed value, and computes a combination of the challenge and the secret key, hidden by the committed mask (the scheme is recalled in more details in Sec. 2.2). The verifier accepts the signature if it satisfies some property (small Euclidean norm) and the verifier did use the challenge.

Recently, Persichetti proposed an efficient (using quasi-cyclic codes) adaptation of this scheme with two major differences: the underlying hard problem is different and there is no rejection sampling. The underlying problem is the renowned Syndrome Decoding (SD) problem, which has been proved NP-hard [2]. The secret key is a vector of small Hamming weight, and the public key is the syndrome of this vector by a public (random) parity-check matrix. According to the author, rejection sampling is not necessary since the signature only depends on the message, the commitment and the challenge (meaning not the secret key).

One of the most technical aspects in the design of a signature scheme is to make the signature distribution statistically independent from the secret key. This allows (by programming the random oracle in the security reduction) the forger to produce valid signatures without knowing the secret key, which can then be used to solve the underlying hard problem. This technicality provides guidance for the choice of the parameters, especially for the Hamming weight (or $\ell_{1}$ norm for Lyubashevsky) of the challenge. Indeed, in order for SD problem to admit a unique solution, the weight of the signature must be below the Gilbert-Varshamov bound. In the meantime, the weight of the secret key should be big enough in order not to be exhibited easily. This implies that the challenge should have an exceptionally low weight for the signature scheme to work. This is indeed the case for all the proposed parameters: the "biggest" (least sparse) challenge has weight $\delta=10$ for length $n=4801$.

From a cryptanalytic point of view, a signature can be rewritten as a noisy decoding problem with known generator matrix: the cyclic matrix obtained through the challenge. Roughly speaking, signatures can be viewed as McEliece encryptions of the secret key under public unscrambled sparse generator matrix. Using such an approach, the matrix corresponds to a Low/Moderate Density ParityCheck (LDPC / MDPC) code. We show that it is possible to use the extended Bit Flipping $(\mathrm{xBF})$ algorithm $[5,9,1]$ to decrypt these ciphertexts, hence retrieving both the secret key and one-time randomness from a single signature, for all the proposed parameters.

Conceptually speaking, the cryptanalysis is possible because the Hamming weight of the challenge is way too small. Increasing this weight would require to lower the weight of the secret key, opening the door for other small weight codeword finding attacks.

Contributions. In this work, we provide evidences that a direct translation of Lyubashevsky's framework to build signatures without trapdoors from lattice assumptions to coding theory assumptions can only yield insecure signatures. It was suspected [12] that such signatures could not reach full-time security due to a statistical bias of the information leaked by the signature. As explained above, the information leakage mostly comes from the sparsity of the challenge vector. As to illustrate our claim, we propose a full cryptanalysis of all the parameters of 
Persichetti's OTS scheme based upon an adaptation of Lyubashevsky's framework. As an example, our attack recovers the signing key of the most secure instance $(n=$ 9857,128 bits of security) in $\approx 450 \mathrm{~ms}$ (versus $100 \mathrm{~ms}$ for signature verification).

Techniques. To conduct a full-cryptanalysis of efficient code based signatures without trapdoors, we begin by formulating the signature cryptanalysis as a decoding problem. The decoding involves a relatively sparse generator matrix (similar to LDPC or MDPC codes). To do so, the signature is split into two halves.

The secret key $\boldsymbol{x}=\left(\boldsymbol{x}_{0}, \boldsymbol{x}_{1}\right)$ has a global weight of $w_{1}$, meaning that $w t\left(\boldsymbol{x}_{0}\right)+$ $w t\left(\boldsymbol{x}_{1}\right)=w_{1}$. But for the cryptanalysis, no hint is provided about the weight of each part, and the same holds for the one-time randomness $\boldsymbol{y}=\left(\boldsymbol{y}_{0}, \boldsymbol{y}_{1}\right)$ used for signing. Therefore, we relax the requirements for decoding the first instance, and reverberate on the second instance using the solutions of the first problem. Once the instances are set up, the $\mathrm{xBF}$ algorithm is used to efficiently solve both instances.

Related works. In an independent work, Santini et al. also proposed a cryptanalysis of Persichetti's OTS [14]. While their attack exploits the same design weakness (namely the sparsity of the challenge), they resort to a statistical analysis on the signature to approximate the secret key $\boldsymbol{x}$. This approximation allows the authors to complete the cryptanalysis by running an Information Set Decoding (ISD) algorithm. On the positive side, the authors do not report failure of their cryptanalysis, whereas the $\times \mathrm{BF}$ decoding algorithm has a non-negligible probability of failing. Fortunately, failures are very unlikely for MDPC codes $\left(\sim 10^{-7}\right)$ and even less likely for LDPC codes and parameters considered in [12] $\left(w \approx n^{1 / 4}\right)$ are much closer to LDPC parameters than MDPC parameters $\left(w \approx n^{1 / 2}\right)$. Additionally, it is sufficient for an attack against a scheme $S$ to work with non-negligible probability to break $S$ with significant advantage. From an efficiency point of view, their attack requires a syndrome computation (considered negligible in [14]) plus an ISD whose complexity heavily depends on the quality of their initial approximation. The attack we propose only requires two syndrome computations (asymptotically, the Bit Flipping algorithm has a complexity linear in the length $n$ of the code). Santini et al. provide an ISD worst-case complexity for a successful attack, that is above $2^{35}$ elementary operations (and asymptotically at least sub-exponential in $n)$, undubiously more expensive than another syndrome computation.

Roadmap. The remainder of this paper is organized as follows: Sec. 2 introduces the notations used throughout this work as long as relevant notions in coding theory and Lyubashevsky's signature scheme. Sec. 3 presents a general adaptation of Lyubashevsky's framework to coding theory, not restricted to specific (quasicyclic) codes. Sec. 4 is devoted to expressing key recovery from a single signature as a decoding problem, and arguing that this problem is efficiently solvable. A general purpose algorithm to solve the latter problem is presented in Sec. 5. We finally instantiate the key recovery with Persichetti's OTS in Sec. 6, presenting a full cryptanalysis, before concluding in Sec. 7 .

Acknowledgement. The authors are grateful to the WCC 2019 and DCC reviewers for their careful reading and relevant comments that helped improving the quality of the present work. 


\section{Preliminaries}

2.1 Notations and definitions

Throughout the paper, $\mathbb{F}_{2}$ denotes the binary field. Vectors (resp. matrices) will be represented in lower-case (resp. upper-case) bold letters, and are row represented. A vector $\boldsymbol{u}=\left(u_{0}, \ldots, u_{n-1}\right) \in \mathbb{F}_{2}^{n}$ will be interchangeably seen as a vector or polynomial in $\mathbb{F}_{2}[x] /\left\langle x^{n}-1\right\rangle$. Hence for $\boldsymbol{u}, \boldsymbol{v} \in \mathbb{F}_{2}^{n}, \boldsymbol{w}=\boldsymbol{u} \boldsymbol{v}$ denotes the vector such that:

$$
w_{k}=\sum_{i+j=k} u_{i} v_{j} x^{k}, \text { for } k \in\{0, \ldots, n-1\} .
$$

Finally, the set of binary vectors of length $n$ and weight exactly $w$ is denoted $\mathcal{S}_{w}^{n}\left(\mathbb{F}_{2}\right)$. We now recall some basic definitions and facts about coding theory that will be helpful for the comprehension of Persichetti's OTS and its cryptanalysis.

Definition 1 (Parity-check matrix) Let $n, k$ be integers. The parity-check matrix of an $[n, k]$ linear code $\mathcal{C}$ is a matrix $\boldsymbol{H} \in \mathbb{F}_{2}^{(n-k) \times n}$ that generates the dual code $\mathcal{C}^{\perp}$. Formally, if $\boldsymbol{G} \in \mathbb{F}_{2}^{k \times n}$ is a generator matrix of $\mathcal{C}$, then $\boldsymbol{H}$ satisfies $\boldsymbol{G} \boldsymbol{H}^{\top}=\mathbf{0}$.

Definition 2 (Syndrome Decoding problem) Let $\boldsymbol{H} \in \mathbb{F}_{2}^{(n-k) \times n}$ be a paritycheck matrix of some $[n, k]$ linear code over $\mathbb{F}_{2}$, and $s \in \mathbb{F}_{2}^{n-k}$ a syndrome, and $w$ an integer. The Syndrome Decoding problem asks to find a vector $\boldsymbol{e} \in \mathbb{F}_{2}^{n}$ of weight less than or equal to $w$ such that $\boldsymbol{s}^{\top}=\boldsymbol{H} \boldsymbol{e}^{\top}$.

The SD problem has been proved to be NP-hard [2]. Assuming a solution to the SD problem exists, the target weight $w$ determines whether the solution can be unique or not. This property is captured through the well-known GilbertVarshamov (GV) bound.

Definition 3 (Gilbert-Varshamov bound) Let $\mathcal{C}$ be an $[n, k]$ linear code over $\mathbb{F}_{q}$. The Gilbert-Varshamov bound $d_{\mathrm{GV}}$ is the maximum value $d$ such that

$$
\sum_{i=0}^{d-1}\left(\begin{array}{l}
n \\
i
\end{array}\right)(q-1)^{i} \leq q^{n-k}
$$

This bound is used in the security proof of Persichetti [12], hence providing guidance for setting the parameters of the OTS. This results in a challenge of exceptionally low weight, that as we show hereafter can be used to retrieve the secret key efficiently.

\subsection{Lattice signatures without trapdoors}

We now recall Lyubashevsky's signature scheme. We keep the description in its general form but as mentioned by the author, key sizes can be shrunk by a factor $k$ using more structured matrices and relying on the ring version of the SIS problem. Also notice that in lattice-based cryptography, vectors are column represented, so in order to keep the same presentation as in the original paper, we deviate from our row-vector representation in this subsection. 
Private and public keys are respectively uniformly random matrices $\boldsymbol{S} \in$ $\{-d, \ldots, 0, \ldots, d\}^{m \times k}$ and $\boldsymbol{A} \in \mathbb{F}_{2}^{n \times m}(\boldsymbol{T}=\boldsymbol{A} \boldsymbol{S}$ also belongs to pk $)$ and the signature process invokes a hash function $\mathcal{H}_{\kappa}:\{0,1\}^{*} \rightarrow\left\{\boldsymbol{v} \in\{0,1\}^{k},\|\boldsymbol{v}\|_{1} \leq \kappa\right\}$. A signature $(\boldsymbol{z}, \boldsymbol{c})$ of a message $\boldsymbol{m}$ corresponds to a combination of the secret key and the hash of this message, shifted by a committed value also used in the hash function. The signature and verification procedures are summarized in Figure 1, the reader is referred to the original paper for full details [8].

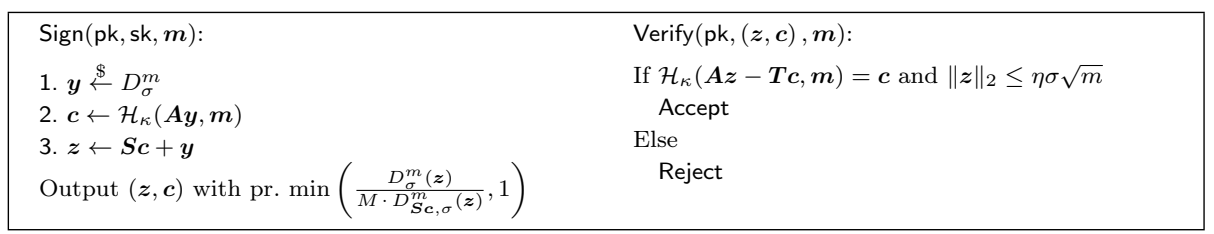

Fig. 1 Sketch of Lyubashevsky's (lattice-based) signature scheme.

Above, $D_{\sigma}$ denotes the discrete Gaussian distribution centered at 0 and of standard deviation $\sigma$. The parameters $\sigma, \eta$, and $\kappa$ are threshold parameters specific to [8] and won't be used hereafter.

\section{Code-based signatures without trapdoors}

In this Section we describe two general code-based adaptations of Lyubashevsky's signature scheme, not restricted to quasi-cyclic codes: a vectorial one, similar to Persichetti's OTS, and a matrix version. The aim of this description is to demonstrate that the weakness of such an adaptation comes from the vectorial version, not from the additional structure added for efficiency. We discuss about the relative (un)security of the matrix adaptation at the end of this section. Both adaptations require a hash function that outputs pseudorandom words of length $n$ and small weight $\delta$. Formally, we denote such a function $\mathcal{H}_{n}:\{0,1\}^{*} \rightarrow \mathcal{S}_{\delta}^{n}\left(\mathbb{F}_{2}\right)$.

A vectorial adaption. This version is a generalization of Persichetti's OTS to not just quasi-cyclic codes. In this vectorial version, the secret key is a vector $\boldsymbol{x}$ of small weight $w_{1}$, and the public key is a random parity-check matrix $\boldsymbol{H}$ together with the syndrome of the secret key: $\boldsymbol{s}_{\boldsymbol{x}}^{\top}=\boldsymbol{H} \boldsymbol{x}^{\top}$. (In Persichetti's proposal, $\boldsymbol{H}$ admits a quasi-cyclic systematic representation: $\boldsymbol{H}=\left(\begin{array}{ll}\mathbf{1} & \boldsymbol{h}\end{array}\right)$, allowing to reduce the pk size.) This adaptation is summarized in Figure 2.

\begin{tabular}{|ll|}
\hline Sign $(\mathrm{pk}, \mathrm{sk}, \boldsymbol{m}):$ & Verify $(\mathrm{pk},(\boldsymbol{z}, \boldsymbol{c}), \boldsymbol{m}):$ \\
1. $\boldsymbol{y} \stackrel{\$}{\leftarrow} \mathcal{S}_{w_{2}}^{n}\left(\mathbb{F}_{2}\right)$ & If $\mathcal{H}_{n}\left(\boldsymbol{z} \boldsymbol{H}^{\top}-\boldsymbol{s}_{\boldsymbol{x}} \boldsymbol{c}, \boldsymbol{m}\right)=\boldsymbol{c}$ and $w t(\boldsymbol{z}) \leq w=\delta w_{1}+w_{2}$ \\
2. $\boldsymbol{c} \leftarrow \mathcal{H}_{n}\left(\boldsymbol{y} \boldsymbol{H}^{\top}, \boldsymbol{m}\right)$ & Accept \\
3. $\boldsymbol{z} \leftarrow \boldsymbol{c} \boldsymbol{x}+\boldsymbol{y}$ & Else \\
Output $(\boldsymbol{z}, \boldsymbol{c})$ & Reject \\
\hline
\end{tabular}

Fig. 2 Description of a code-based vectorial adaptation of Lyubashevsky's framework. 
To sign a message $\boldsymbol{m}$, a mask $\boldsymbol{y}$ of small weight $w_{2}$ is sampled uniformly at random, then committed by its syndrome, together with the message, to get the challenge $\boldsymbol{c}=\mathcal{H}_{n}\left(\boldsymbol{y} \boldsymbol{H}^{\top}, \boldsymbol{m}\right)$. The response to this challenge is the polynomial product of the secret key and the challenge, hidden by the committed mask: $\boldsymbol{z}=$ $\boldsymbol{c} \boldsymbol{x}+\boldsymbol{y}$. The signature consists of the challenge and the response: $\sigma=(\boldsymbol{z}, \boldsymbol{c})$.

Matrix version. We now describe a generalization of Persichetti's OTS to matrices. Our generalization is actually closer to Lyubashevsky's original work [8] for general lattices, not just ideals. It is also more connected to the SD problem in some sense since the response computation involves a syndrome computation instead of just a polynomial multiplication. Yet while this generalization permits to avoid the full cryptanalysis directly from one signature, it still leaks some information that reveals the secret key within a few signatures. Actually, this construction is similar to a submission to NIST post-quantum standardization process ${ }^{1}$ named RaCoSS [10]. One of the main difference with this proposal lies in the distribution of the secret key rows (probabilistic vs deterministic). RaCoSS has already been attacked [3], then patched [11], then attacked again [15].

The secret key consists of $m$ vectors $\boldsymbol{x}_{0}, \ldots, \boldsymbol{x}_{m-1}$ of small weights $w_{1}$, that constitute the row of the private matrix $\boldsymbol{X} \in \mathbb{F}_{2}^{m \times n}$. As in the previous subsection, the public key is a random parity-check matrix $\boldsymbol{H} \in \mathbb{F}_{2}^{(n-k) \times n}$ (not necessarily quasicyclic) together with the syndromes of the secret key $\boldsymbol{S}^{\top}=\boldsymbol{H} \boldsymbol{X}^{\top} \in \mathbb{F}_{2}^{(n-k) \times m}$. This adaptation is summarized in Figure 3.

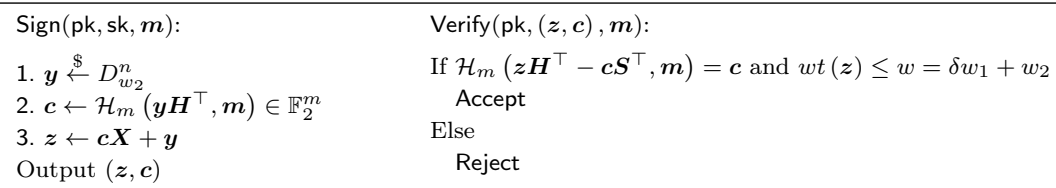

Fig. 3 Description of a code-based matricial adaptation of Lyubashevsky's framework.

The main difference between the vector and matrix versions lies in the signature computation. Indeed, while the first steps are identical, the response computation is pretty different. It resembles more a McEliece encryption of "message" $\boldsymbol{c}$, with generator matrix $\boldsymbol{X}$ and error $\boldsymbol{y}$. However, the message $\boldsymbol{c}$ is public here, while matrix $\boldsymbol{X}$ is not. Yet this does not prevent information leakage, and the secret key can still be recovered using a limited number of signatures as exhibited by attacks on RaCoSS [3, 15].

Information leakage and rejection sampling. The most important (and costly) step in Lyubashevsky's full-time signature scheme is the final one: rejection sampling. This step is performed before publishing any signature to ensure that the candidate response $\boldsymbol{z}=\boldsymbol{S} \boldsymbol{c}+\boldsymbol{y}$ does not leak any information about the secret key. In other words, it enforces the signature distribution to be statistically (or at least computationally) independent from the secret key. As mentioned before, this is done to let an adversary against the existential unforgeability under

\footnotetext{
1 See https://csrc.nist.gov/projects/post-quantum-cryptography.
} 
chosen message attack (EUF-CMA for short) successfully produce a valid signature without knowing the secret key, in order to then exploit this forgery to solve the underlying hard problem (namely SIS for [8]).

This has for main consequence that the candidate response is output only with some probability smaller than one. Persichetti's OTS does not use rejection sampling at all. This is probably done in the hope that the information leaked in the OTS is not sufficient to retrieve the secret key. We show in the next sections that the leak is inherent to the signature design and the difference between Hamming and Euclidean metrics, and not due to the lack of rejection sampling.

\section{One-time signature as a decoding problem}

In this section, we focus on the vector adaptation to rewrite the cryptanalytic problem as a decoding problem. Recall that in (the general version of) Persichetti's OTS, the signature is a couple $(\boldsymbol{z}, \boldsymbol{c})$ with $\boldsymbol{z}=\boldsymbol{c x}+\boldsymbol{y}, w t(\boldsymbol{x})=w_{1}, w t(\boldsymbol{y})=w_{2}$ and $w t(\boldsymbol{c})=\delta$ so that $w t(\boldsymbol{z}) \leq w=\delta w_{1}+w_{2}$. The author claims [12, Sec. 4 p. 6]:

" $A$ big advantage of our proposal is that this issue (introducing extra algebraic structure can compromise the secrecy of the private matrix used for decoding) does not apply. In fact, since there is no decoding involved, an entirely random code can be used, and the code itself is public, so there is no private matrix to hide. In this sense, our scheme is closer, to an extent, to the work of [1], which is centered on random quasi-cyclic codes."

We show that this statement is not accurate, and that the problem of recovering the secret key (and one time randomness) from the OTS can indeed involve decoding. Polynomial multiplication in $\mathbb{F}_{2}[x] /\left\langle x^{n}-1\right\rangle$ can be interchangeably seen as a matrix-vector multiplication in $\mathbb{F}_{2}^{n \times n} \times \mathbb{F}_{2}^{n}$. To do so, we use the following notation: for a vector $\boldsymbol{v}=\left(v_{0}, \ldots, v_{n-1}\right) \in \mathbb{F}_{2}^{n}$, we denote by $\operatorname{rot}(\boldsymbol{v})$ the cyclic matrix obtained using the cyclic right shifts of $\boldsymbol{v}$. Formally:

$$
\operatorname{rot}(\boldsymbol{v})=\left(\begin{array}{cccc}
v_{0} & v_{n-1} & \ldots & v_{1} \\
v_{1} & v_{0} & \ldots & v_{2} \\
\vdots & \vdots & \ddots & \vdots \\
v_{n-1} & v_{n-2} & \ldots & v_{0}
\end{array}\right) \in \mathbb{F}_{2}^{n \times n}
$$

Using the notation above, any polynomial multiplication $\boldsymbol{a} \times \boldsymbol{b}$ can now be written $\operatorname{rot}(\boldsymbol{a}) \boldsymbol{b}^{\top}$ (resulting in a column vector). We can apply this rewriting to line 3. of the signature algorithm (we re-introduce the $\operatorname{transpose}^{\top}$ notation to be consistent with the row representation):

$$
\boldsymbol{z}^{\top}=(\boldsymbol{c x})^{\top}+\boldsymbol{y}^{\top}=\operatorname{rot}(\boldsymbol{c}) \boldsymbol{x}^{\top}+\boldsymbol{y}^{\top}
$$

Due to the constraint mentioned in the previous section, namely the GV bound, $c$ has to be of particularly low weight $\delta$. To give an idea of the order of magnitude, if $n$ is the length of the code being used, the challenge should have weight approximately $\delta \in \mathcal{O}\left(n^{1 / 4}\right)$ for the signature to be unique. This implies in particular that the matrix rot $(\boldsymbol{c})$ is sparse, and defines an LDPC or MDPC code $\mathcal{C}$. 
From a cryptanalytic point of view, we have that the response $\boldsymbol{z}$ in the signature is equal to the syndrome of the secret key $\boldsymbol{x}$ by the sparse matrix rot $(\boldsymbol{c})$, hidden by a random error $\boldsymbol{y}$ of small weight $w_{2}$. But the challenge $\boldsymbol{c}$ is part of the signature so that any adversary $\mathcal{A}$ against the EUF-CMA of the scheme has access to $c$ (and hence $\operatorname{rot}(\boldsymbol{c}))$. Therefore, to recover the secret key $\boldsymbol{x}$ (and one time randomness $\boldsymbol{y}), \mathcal{A}$ is left with a noisy version of the syndrome decoding problem, involving a public MDPC code, which contradicts Persichetti's claim as stated. We now present an efficient algorithm to solve this decoding problem.

\section{Extended Bit Flipping algorithm}

In this Section, we briefly describe a simple xBF algorithm version. The bit flipping algorithm was originally introduced by Gallager [5] to decode LDPC codes. It later proved to be much more versatile, allowing to efficiently decode MDPC codes [9], even with noisy syndromes [4]. It is actually a natural approach for decoding: using the fact that every codeword has a null syndrome, the algorithm aims at reducing the number of unsatisfied parity-check equations at each iteration. By maximum likelihood, a bit $x_{i}$ of $\boldsymbol{x}$ is flipped if it allows to reduce more than a certain number (threshold) $\tau$ of unsatisfied parity check equations $s_{j}=\boldsymbol{H}_{j} \boldsymbol{x}^{\top}$ (with $\boldsymbol{H}_{j}$ the row of $\boldsymbol{H}$ indexed by $j$ ). The algorithm stops when the updated syndrome is null, or has weight less than some bound for the noisy version (the algorithm can also fail and stop after a predefined maximum number $N$ of iterations). The complete $\times B F$ algorithm is described in Algo. 1.

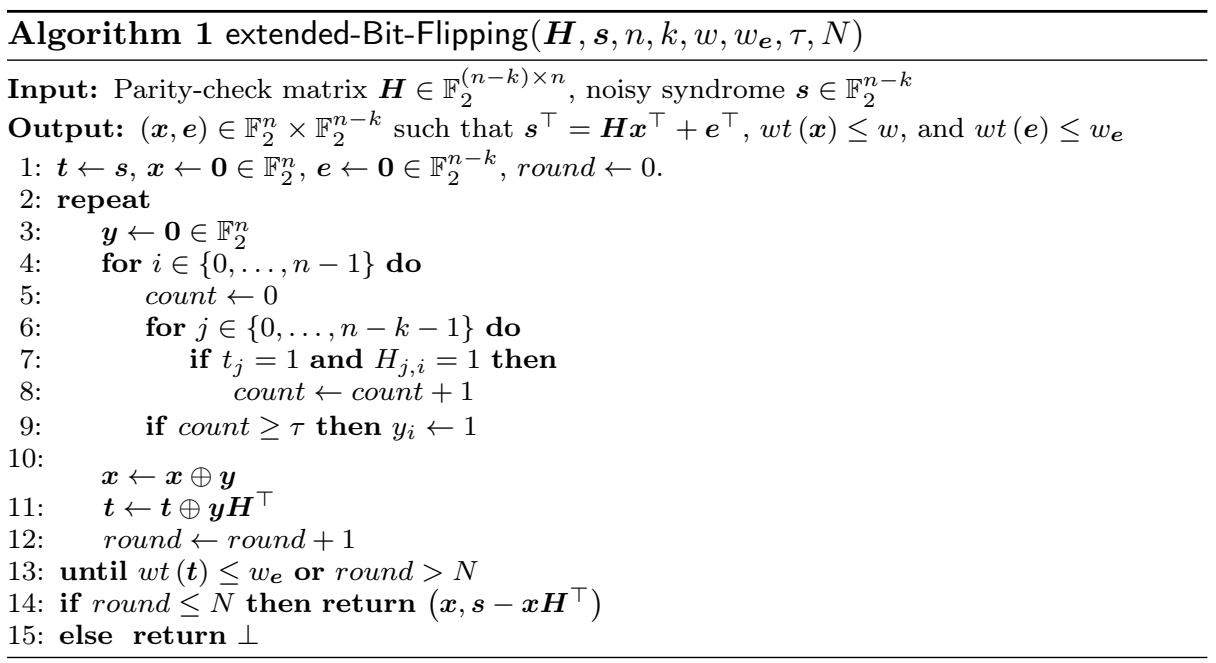

We are now equipped with all the tools to perform the full cryptanalysis of the (generalization of the) efficient OTS of Persichetti. 


\section{Full cryptanalysis of Persichetti's one time signature scheme}

In this section, we put the previous pieces together and report a full cryptanalysis of Persichetti's OTS. We show that it is possible to recover the secret key (and hence the one time randomness used for signing too) from a single signature in less than a second, for all the proposed parameters. The cryptanalysis is summarized in Algo. 2. Persichetti uses a special ring instantiation to try to add more confusion to the signature. Let $n=2 p$. In Persichetti's scheme, the secret key consists of $\boldsymbol{x}=\left(\boldsymbol{x}_{0}, \boldsymbol{x}_{1}\right) \in \mathbb{F}_{2}^{p} \times \mathbb{F}_{2}^{p}$ of global weight $w_{1}$, meaning that $w t\left(\boldsymbol{x}_{0}\right)+w t\left(\boldsymbol{x}_{1}\right)=w_{1}$. A signature is a couple $(\boldsymbol{z}, \boldsymbol{c}) \in \mathbb{F}_{2}^{n} \times \mathbb{F}_{2}^{p}$ with $\boldsymbol{z}=\left(\boldsymbol{z}_{0}, \boldsymbol{z}_{1}\right)$ and $\boldsymbol{z}_{i}=\boldsymbol{x}_{i} \boldsymbol{c}+\boldsymbol{y}_{i}$, such that $w t(\boldsymbol{z})=w t\left(\boldsymbol{z}_{0}\right)+w t\left(\boldsymbol{z}_{1}\right) \leq w=\delta w_{1}+w_{2}$, and $w t(\boldsymbol{c}) \leq \delta \approx n^{1 / 4}$. The one-time randomness $\boldsymbol{y}=\left(\boldsymbol{y}_{0}, \boldsymbol{y}_{1}\right) \in \mathbb{F}_{2}^{p} \times \mathbb{F}_{2}^{p}$ has global weight $w_{2}$. The goal of the cryptanalysis is to retrieve $\boldsymbol{x}_{0}$ and $\boldsymbol{x}_{1}$ from $\boldsymbol{z}$ and $\boldsymbol{c}$.

The first step of the cryptanalysis is to decompose the target into two halves: $\boldsymbol{z}_{0}=\boldsymbol{x}_{0} \boldsymbol{c}+\boldsymbol{y}_{0}$ and $\boldsymbol{z}_{1}=\boldsymbol{x}_{1} \boldsymbol{c}+\boldsymbol{y}_{1}$. Each equation of this system can then be viewed independently as a noisy syndrome decoding problem, with public MDPC matrix rot $(\boldsymbol{c})$ as explained in Sec.4. Using the XBF algorithm described in Sec. 5, one can solve each line of the system ( $\tau$ and $N$ will be specified later in this Section):

$$
\left(\boldsymbol{x}_{i}, \boldsymbol{y}_{i}\right) \leftarrow \text { extended-Bit-Flipping }\left(\boldsymbol{\operatorname { r o t }}(\boldsymbol{c}), \boldsymbol{z}_{i}, n, p, w_{1} / 2, w_{2} / 2, \tau, N\right) .
$$

A tiny technical caveat needs to be handled for breaking the scheme in practice: the repartition of the noise. Indeed, while the global weight of $\boldsymbol{x}$ (resp. $\boldsymbol{y}$ ) is $w_{1}$ (resp. $w_{2}$ ), it is unlikely to always have $w t\left(\boldsymbol{x}_{0}\right)=w t\left(\boldsymbol{x}_{1}\right)=w_{1} / 2$ and $w t\left(\boldsymbol{y}_{0}\right)=$ $w t\left(\boldsymbol{y}_{1}\right)=w_{2} / 2$. We therefore introduce a relaxation parameter: the integer relax $\epsilon$ $\left\{0, \ldots, \min \left(w_{1}, w_{2}\right)\right\}$, and will allow the weight of the candidate solution $\tilde{\boldsymbol{x}}_{i}$ and $\tilde{\boldsymbol{y}}_{i}$ to be respectively within $w_{1} / 2 \pm$ relax and $w_{2} / 2 \pm$ relax. Experimentally, setting relax $=w_{2} / 4=\max \left\{w_{1}, w_{2}\right\} / 4$ provides satisfactory results for the cryptanalysis.

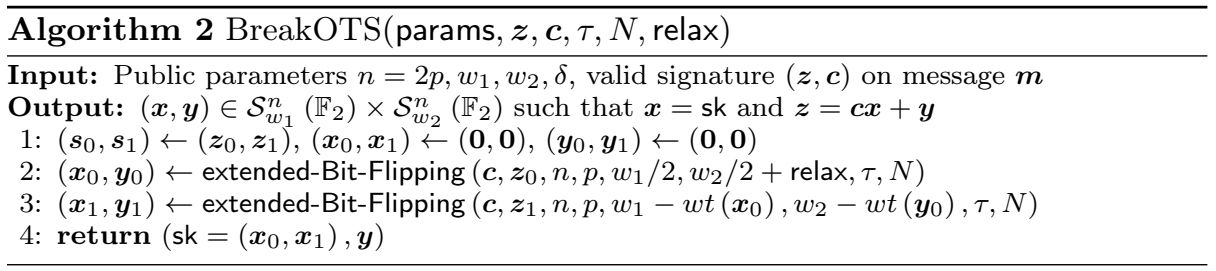

Finally, a basic implementation of the cryptanalysis is available at https: //github.com/deneuville/PersichettiOTScryptanalysis. The code was compiled using GCC 5.4.0 using flags -std=c++11 -fpermissive -03, and run on a single Intel ${ }^{\circledR}$ Core $^{\text {TM }}$ i7-6920HQ CPU @ 2.90GHz with TurboBoost disabled. The timings reported in Table 1 are expressed in milliseconds. The verification timings come from the original paper [12]. While they were obtained on a seemingly less powerful device, they compare favorably to our highly unoptimized proof of concept implementation of Persichetti's OTS. Therefore we conservatively chose to refer to these timings.

Notice that Algo. 2 is presented using quasi-cyclic codes: the parity-check matrix given to the $\times \mathrm{BF}$ algorithm consists of the cyclic shift of vector $c$. The cryptanalysis timings reported in Table 1 correspond to a generic version of the $x B F$ 
Table 1 Parameters for Persichetti's OTS (from [12]) and for the xBF algorithm. All timings are in milliseconds. The timings for the cryptanalysis roughly correspond to two $\times B F$ runs (one for each part of the secret key). The verification timings were taken directly from [12]. The last $\times \mathrm{BF}$ parameter relax (not shown in this table) is always set to $w_{2} / 4$.

\begin{tabular}{|c|c|c|c|c|c|c|c|c|}
\hline \multirow[b]{2}{*}{ security } & \multicolumn{4}{|c|}{ Persichetti's OTS parameters } & \multicolumn{2}{|c|}{ xBF parameters } & \multirow{2}{*}{$\begin{array}{l}\text { Verification } \\
t_{\text {verify }}(\mathrm{ms})\end{array}$} & \multirow{2}{*}{$\begin{array}{c}\text { Cryptanalysis } \\
t_{\text {break }}(\mathrm{ms}) \\
\end{array}$} \\
\hline & $n$ & $w_{1}$ & $w_{2}$ & $\delta$ & $\tau$ & $N$ & & \\
\hline \multirow{2}{*}{80} & 4801 & 90 & 100 & 10 & 7 & 5 & 22.569 & 165.459 \\
\hline & 3072 & 85 & 85 & 7 & 5 & 5 & 14.271 & 68.858 \\
\hline \multirow{2}{*}{128} & 9857 & 150 & 200 & 12 & 9 & 10 & 99.492 & 453.680 \\
\hline & 6272 & 125 & 125 & 10 & 7 & 10 & 42.957 & 288.442 \\
\hline
\end{tabular}

algorithm. Due to the very peculiar structure of the parity-check matrix (cyclic and sparse), it is actually possible to optimize much more the $\times B F$ algorithm. Persichetti's verification requires one syndrome computation: $\boldsymbol{s}_{\boldsymbol{z}}=\boldsymbol{z}_{0}+\boldsymbol{h} \boldsymbol{z}_{1}$, equivalent to one full-sparse polynomial multiplication and one addition, a sparse-full polynomial multiplication: $\boldsymbol{c} \boldsymbol{s}_{\boldsymbol{x}}$, and another polynomial addition: $\boldsymbol{c s}_{\boldsymbol{x}}+\boldsymbol{s}_{\boldsymbol{z}}$. An xBF essentially corresponds to a syndrome computation, plus some polynomial additions on positions flipped during execution. Therefore, an optimized xBF algorithm taking advantage from this cyclic and sparse structure would require one syndrome computation: $\boldsymbol{c} \tilde{\boldsymbol{x}}_{b}(b \in\{0,1\}), \tilde{\boldsymbol{x}}_{b}$ being the guessed secret, equivalent to one sparse-sparse polynomial multiplication, $w_{1}$ polynomial additions (equivalent to another sparse-sparse polynomial multiplication), and some other overhead polynomial additions and memory access for threshold verification and syndrome updates. Two xBF runs are required for the full cryptanalysis, involving twice as many polynomial multiplications (the most expensive operation) as for the signature verification. This reasonably lets us believe that a fully optimized cryptanalysis implementation should completely break Persichetti's OTS scheme in no longer than twice the verification time.

\section{Conclusion}

In this paper, we have presented an attack on efficient OTS without trapdoors, based on codes (not necessarily quasi-cyclic). This attack targets the vectorial adaptation of Lyubashevsky's signature scheme. Viewing the commitment as an $\mathrm{LDPC} / \mathrm{MDPC}$ code, it is possible to rewrite the signature as a noisy syndrome decoding problem, for which the $\mathrm{xBF}$ algorithm is especially suited. Applied to Persichetti's scheme, we retrieve the secret key in less than a second for all parameters, disproving the claimed 80 to 128 bits security. While the matrix version of this adaptation seems less sensitive to this attack, it clearly leaks information on the support of the secret key, that can be retrieved using a few signatures, as noticed in the original adaptation [12].

\section{References}

1. Aguilar Melchor, C., Blazy, O., Deneuville, J., Gaborit, P., Zémor, G.: Efficient encryption from random quasi-cyclic codes. IEEE Trans. Information Theory 64(5) (2018) 3927-3943 2, 7 
2. Berlekamp, E.R., McEliece, R.J., van Tilborg, H.C.A.: On the inherent intractability of certain coding problems (corresp.). IEEE Trans. Information Theory 24(3) (1978) 384-386 2, 4

3. Daniel Julius, B., Andreas, H., Tanja, L., Panny, L.: OFFICIAL COMMENT: RaCoSS. Official comments about NIST PQC submissions (December 2017) 6

4. Deneuville, J.C., Gaborit, P., Zémor, G.: Ouroboros: A simple, secure and efficient key exchange protocol based on coding theory. In: International Workshop on Post-Quantum Cryptography, Springer (2017) 18-34 8

5. Gallager, R.: Low-density parity-check codes. IRE Transactions on information theory 8(1) (1962) 21-28 2, 8

6. Gentry, C., Peikert, C., Vaikuntanathan, V.: Trapdoors for hard lattices and new cryptographic constructions. In Ladner, R.E., Dwork, C., eds.: 40th ACM STOC, ACM Press (May 2008) 197-206 1

7. Hoffstein, J., Pipher, J., Silverman, J.H.: NSS: An NTRU lattice-based signature scheme. In Pfitzmann, B., ed.: EUROCRYPT 2001. Volume 2045 of LNCS., Springer, Heidelberg (May 2001) 211-228 1

8. Lyubashevsky, V.: Lattice signatures without trapdoors. In Pointcheval, D., Johansson, T., eds.: EUROCRYPT 2012. Volume 7237 of LNCS., Springer, Heidelberg (April 2012) 738-755 1, 5, 6, 7

9. Misoczki, R., Tillich, J.P., Sendrier, N., Barreto, P.S.: Mdpc-mceliece: New mceliece variants from moderate density parity-check codes. In: Information Theory Proceedings (ISIT), 2013 IEEE International Symposium on, IEEE (2013) 2069-2073 2, 8

10. Partha Sarathi, R., Rui, X., Kazuhide, F., Shinsaku, K., Kirill, M., Tsuyoshi, T.: RaCoSS: Random code-based signature scheme. Submission to NIST post-quantum standardization process (November 2017) 6

11. Partha Sarathi, R., Rui, X., Kazuhide, F., Shinsaku, K., Kirill, M., Tsuyoshi, T.: Code-based signature scheme without trapdoors. IEICE Tech. Rep., vol. 118, no. 151, ISEC2018-15, pp. 17-22 (July 2018) https://www.ieice.org/ ken/paper/20180725L1FF/eng/. 6

12. Persichetti, E.: Efficient one-time signatures from quasi-cyclic codes: A full treatment. Cryptography 2(4) (2018) 30 1, 2, 3, 4, 7, 9, 10

13. Pointcheval, D., Stern, J.: Security arguments for digital signatures and blind signatures. Journal of cryptology 13(3) (2000) 361-396 1

14. Santini, P., Baldi, M., Chiaraluce, F.: Cryptanalysis of a one-time code-based digital signature scheme. In: 2019 IEEE International Symposium on Information Theory (ISIT), IEEE (2019) 2594-2598 3

15. Xagawa, K.: Practical attack on racoss-r. Cryptology ePrint Archive, Report 2018/831 (2018) https://eprint.iacr.org/2018/831. 6 